\title{
Rib head disarticulation for multilevel transpedicular thoracic corpectomies and expandable cage reconstruction
}

\author{
Dean Chou, Matthew Eltgroth, Isaac Yang, Daniel Lu, Geoff Manley \\ Department of Neurological Surgery, University of California San Francisco, USA
}

\author{
Address for correspondence: \\ Dr. Dean Chou, \\ Department of Neurological \\ Surgery, University of California, \\ San Francisco, 505 Parnassus Ave. \\ Box 0112 , San Francisco, \\ CA 94143-0112, USA. \\ E-mail: choud@neurosurg.ucsf.edu
}

DOI: $10.4103 / 0028-3886.55602$

\begin{abstract}
Traditional posterior approaches to the thoracic spine are done with either costo-transversectomy, lateral extracavitary approaches, or transpedicular approaches. The transpedicular approach is the only one that preserves the rib head. However, placing an expandable cage with this rib head intact poses special challenges because of the narrow corridor defined by the rib head and the spinal cord. Instead of removing the rib head, which reeuires pleural dissection and carries the risks of pleural injury, we disarticulate it and push it laterally during cage placement. This avoids pleural dissection and affords expandable cage placement through a transpedicular approach.
\end{abstract}

Key words: Cage, corpectomy, costotransversectomy, disarticulation, lateral extracavitary approach, posterior, multilevel, reconstruction, rib head, thoracic, transpedicular

\section{Introduction}

Traditionally, multilevel thoracic corpectomies are done from an anterior approach. This is done for multiple reasons: Easy access to pathology, correction of kyphosis, direct visualization for reconstruction, and direct decompression of the spinal cord. However, many techniques have described posterior corpectomies and reconstruction in the thoracic spine. Multilevel thoracic corpectomies from a posterior approach pose special challenges. Sufficient anterior access, adequate decompression, spinal destabilization, sound reconstruction, and risk of pleural injury are some factors that must be taken into consideration. Costotransversectomy and lateral extracavitary approaches have been well described, but they are associated with rib head removal and risk of pleural injury. ${ }^{[1]}$ We describe a technique which may facilitate multilevel posterior thoracic corpectomies with expandable cage reconstruction and preservation of the rib heads.

\section{Materials and Methods}

\section{Surgical technique}

After identifying the correct levels of pathology by computed tomography (CT) or magnetic resonance imaging (MRI), the degree of kyphosis, the bone quality, and the number of levels to be removed are all taken into consideration. This will dictate how many levels above and below the pathology that the instrumentation should be placed.

Because these corpectomies are very destabilizing, appropriate levels of instrumentation should be taken into account for the stability of the construct. Instrumentation is extended beyond the apex of thoracic kyphosis if the kyphosis is significant. The degree of osteoporosis also needs to be considered when planning the number of levels of instrumentation.

After general anesthesia is induced, the patient is placed prone onto the Jackson table. A preoperative X-ray is taken to localize the appropriate level. After prepping the skin, a midline skin incision is performed. Muscle dissection is carried out laterally to the edges of the transverse processes. An intraoperative $\mathrm{X}$-ray is taken to confirm the level. Thoracic pedicle screws are placed into the levels above and below the planned corpectomies. After this has been done, laminectomies are performed at the level of the pathology. Partial laminectomies above and below these sites are also performed in order to provide full access to the anterior vertebral column. 
The transpedicular corpectomies are begun and the thoracic nerves are ligated. The corpectomies are carried out with a high speed burr, and in order to adequately complete the corpectomy circumferentially, it is useful to ligate the nerve roots bilaterally. However, if the corpectomy can be achieved without nerve root ligation, this should be performed. Usually, one of the nerve roots must be ligated in order to place the expandable cage. The endplates of the levels above and below the pathology are identified and can be confirmed with fluoroscopy. As the corpectomies become more extensive, temporary rods should be placed on one side to prevent translation of the spinal column. After the corpectomies are finished, the junction between the rib and remnant of the vertebral body is identified, usually with electrocautery. A Cobb elevator is placed in-between to disarticulate the rib head from the remaining vertebral body. The Cobb is initially placed between the rib and the vertebral body remnant, gently inserted further with a mallet, and the rib is disarticulated by rotating the Cobb. Because the rib head articulates between two vertebral bodies, the cephalad most rib must also be disarticulated from the cephalad most vertebral body which will be left intact. Any remaining bone left on the lateral wall of the vertebral body is removed completely, and thorough discectomies are performed.
The disarticulation step is repeated through all the levels of corpectomies. This allows gentle retraction of the chest wall laterally without pleural dissection. An appropriate cage is sized and then placed by retracting the chest wall laterally. This can be done with the cage itself or a Cobb elevator, pushing the chest wall laterally as the cage slides ventral to the spinal cord. Once the cage is anterior to the spinal cord, the chest wall retraction can be released. The cage is expanded under fluoroscopy to the appropriate height. If further height restoration is desired, the set screws along the temporary rod can be released. After this is done, the set screws and cage expansion device are all tightened [Figure 1]. A final AP and lateral fluoroscopic radiograph is taken to view the overall construct.

\section{Results}

\section{Case illustrations}

Case 1

A 71-year-old woman presented to an outside institution with difficulty in walking. She had a history of methicillinresistant Staphylococcus aureus bacteremia secondary to a foot ulcer. An MRI showed cord compression, retropulsion of canal fragments, kyphosis, and vertebral body destruction at vertebral bodies T8, 9, and 10

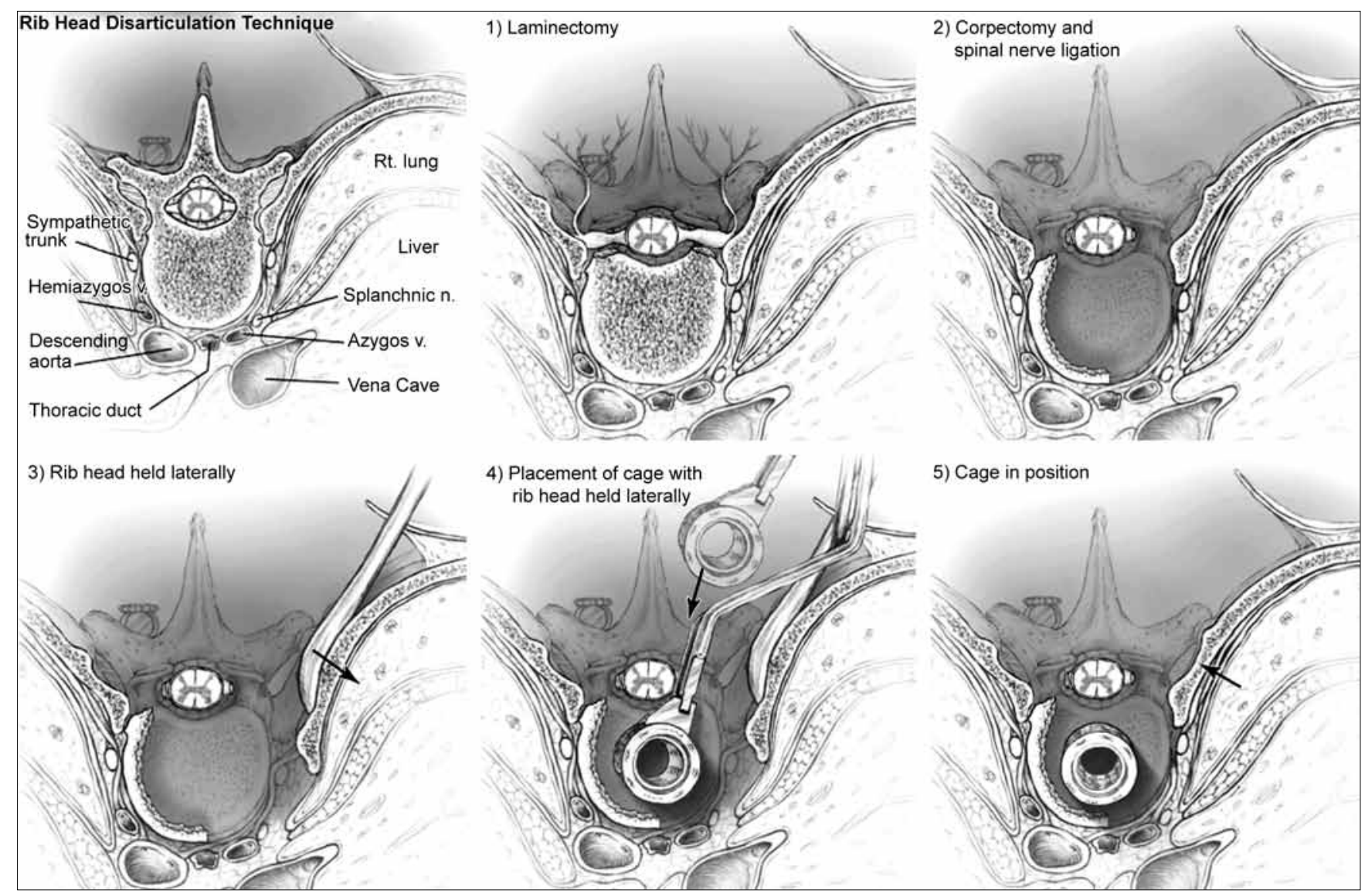

Figure 1: Artist's schematic illustrating technique of rib head disarticulation to facilitate expandable cage placement 
[Figure 2]. Because of pulmonary concerns, she underwent a three-level posterior transpedicular corpectomy and rib head disarticulation to facilitate cage placement (VBR, Ulrich Medical, Germany) [Figure3]. Instrumentation was placed four levels above and below from T4-L2 (Synthes USS, Westchester, PA), and fusion was performed with autologous bone for spinal reconstruction.

\section{Case 2}

An 80-year-old male with a history of tuberculosis presented to our institution with progressive weakness and an inability to ambulate. An MRI showed cord compression, a CT showed vertebral body destruction at T10-11 secondary to Pott's disease [Figure 4], and plain radiographs showed severe kyphosis with sitting [Figure 5]. The patient underwent T10-11 transpedicular corpectomies, rib head disarticulation, and instrumentation with cage reconstruction from T7-L2. The patient had kyphosis correction, and the postoperative CT scan showed good anterior column reconstruction [Figures 6 and 7].

\section{Case 3}

A 69-year-old man with known history of nonsmall cell lung cancer presented with back pain. He was diagnosed by MRI with a T3 metastatic lesion causing cord compression. Neurologic examination demonstrated some numbness below the T3 sensory level, but no weakness. Because he had cord compression and disease within the vertebral body, we performed a T3 transpedicular corpectomy with rib head disarticulation.

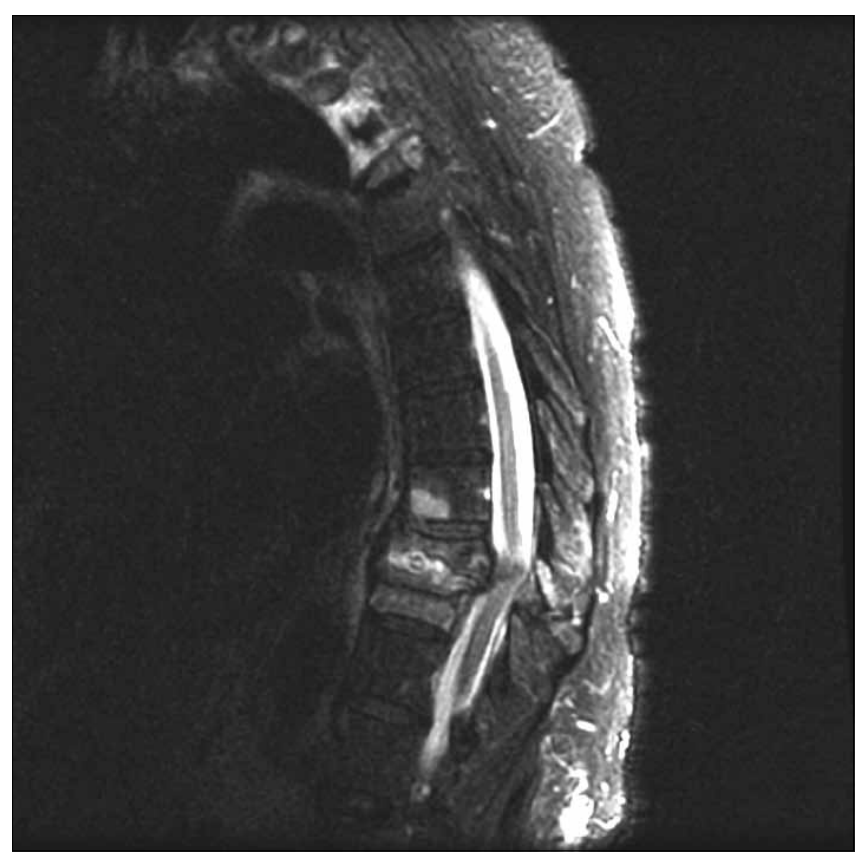

Figure 2: Sagittal T2-weighted MRI showing vertebral body osteomyelitis at T8-10 and cord compression

\section{Surgical outcomes and complications}

The patient in Case 1 developed a postoperative pleural effusion which required a chest tube for drainage. She ultimately did well from a neurologic standpoint with gradual improvement of her neurologic function. The patient in Case 2 did very well from a neurologic standpoint and did not suffer from postoperative complications. The patient in Case 3 did extremely well, with 16-month survival to date after the procedure with intact neurologic function.

\section{Discussion}

Access to anterior thoracic spine pathology has traditionally been performed with an anterior approach via thoracotomy. This allows for direct decompression, reconstruction of the anterior column, height restoration, and kyphosis correction. However, well-documented complications of the thoracotomy include pneumothorax, hemothorax, and vascular injury. ${ }^{[2,3]} \mathrm{A}$ chest tube is also required postoperatively.

Alternatively, a number of posterior approaches have been used to access the anterior vertebral column for the treatment of metastasis, trauma, infection, and kyphosis. ${ }^{[2,4-8]}$

Posterior approaches, including the transpedicular corpectomy, the costotransversectomy, the lateral extracavitary approach, and modifications thereof have been used to access the anterior thoracic spine from a posterior approach. ${ }^{[3,9]}$ Advantages of a single posterior

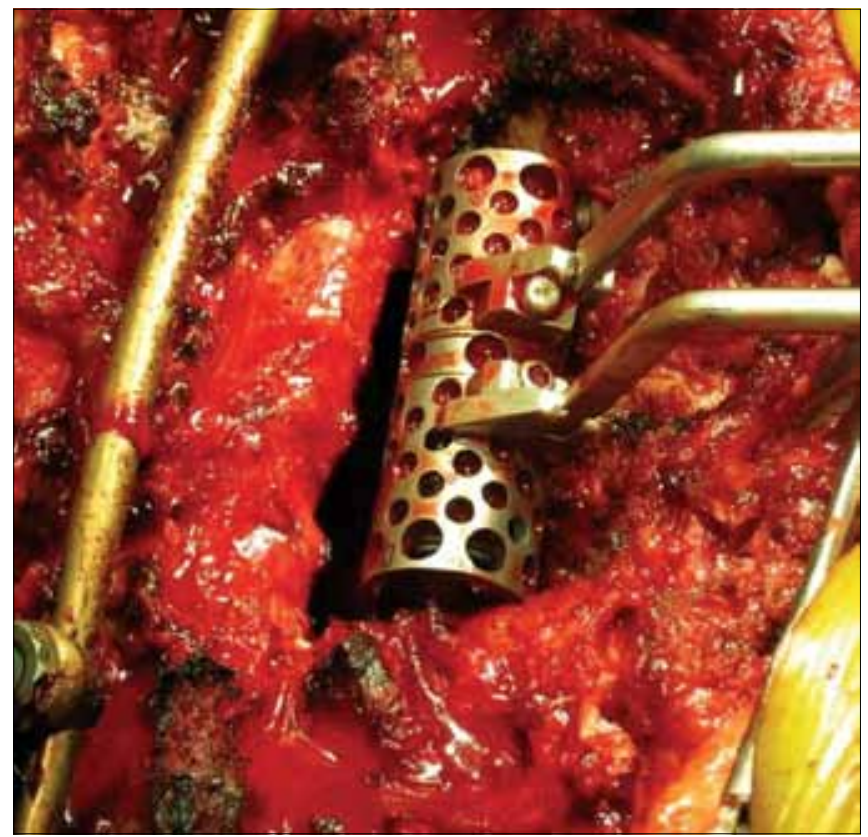

Figure 3: Intraoperative photo showing cage being placed by gently pushing chest wall laterally after rib head disarticulation 


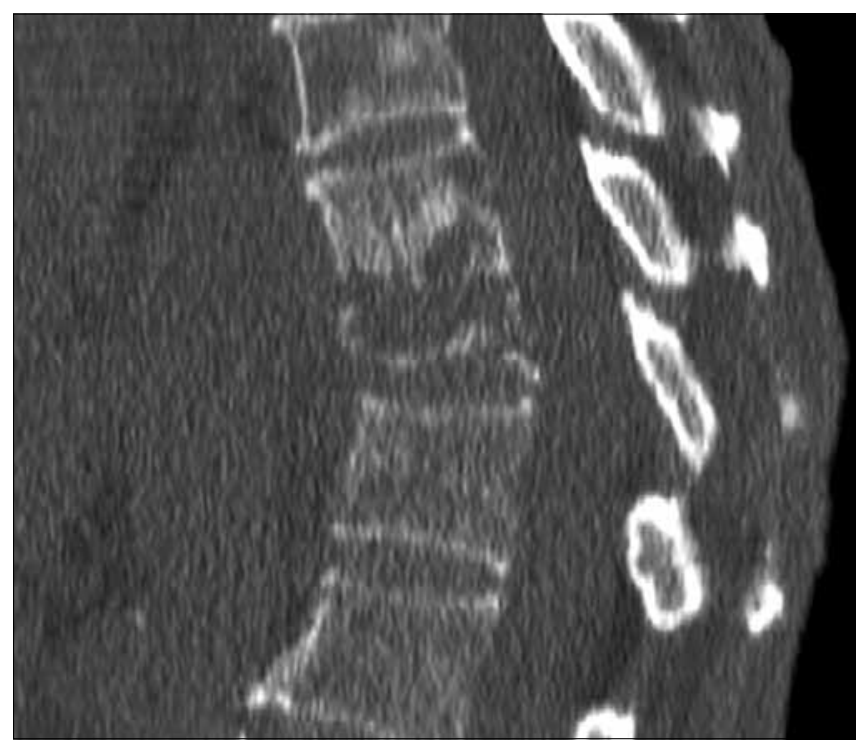

Figure 4: Preoperative CT scan showing T10-11 vertebral body destruction

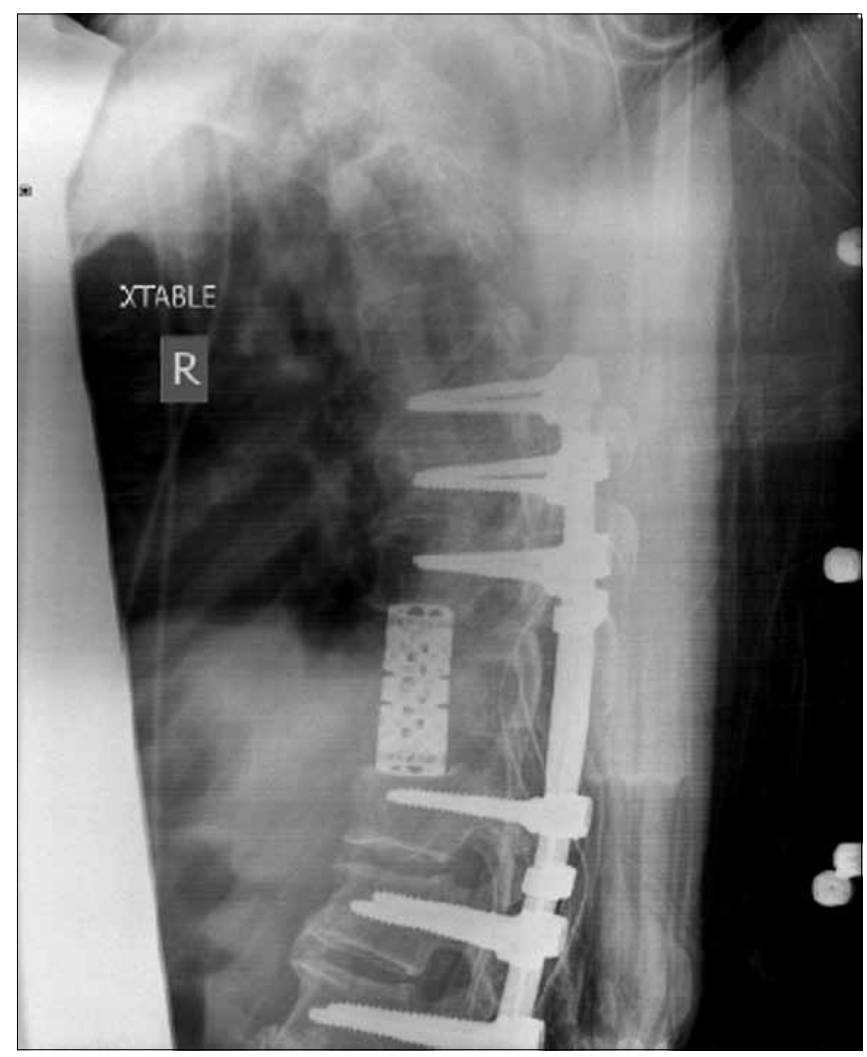

Figure 6: Postoperative lateral radiograph showing cage placement and correction of kyphosis

approach include single-staged surgery, avoidance of a thoracotomy, and potentially a faster recovery period..$^{[10]}$

In most of these clinical series, the posterior approach is performed by either a costotransversectomy or lateral extracavitary approach. In both techniques, rib head resections are required. As a result, such complications as injury to the pleura and neurovascular bundle may occur.

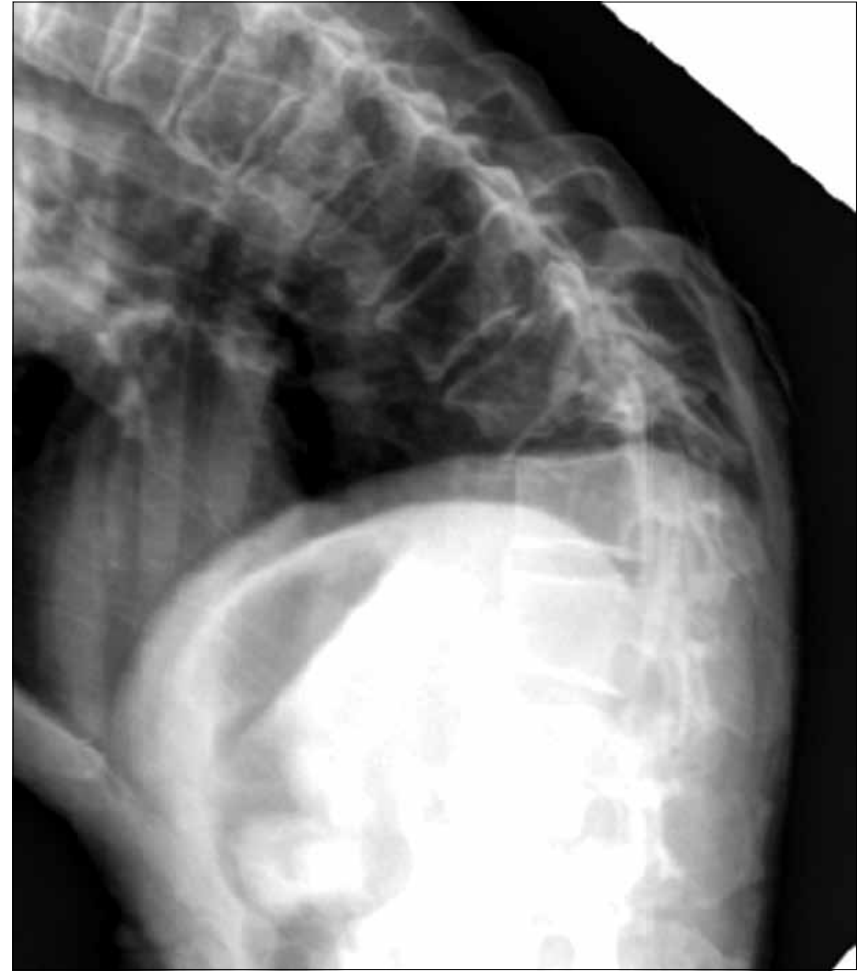

Figure 5: Preoperative lateral radiograph showing severe kyphosis and vertebral body destruction

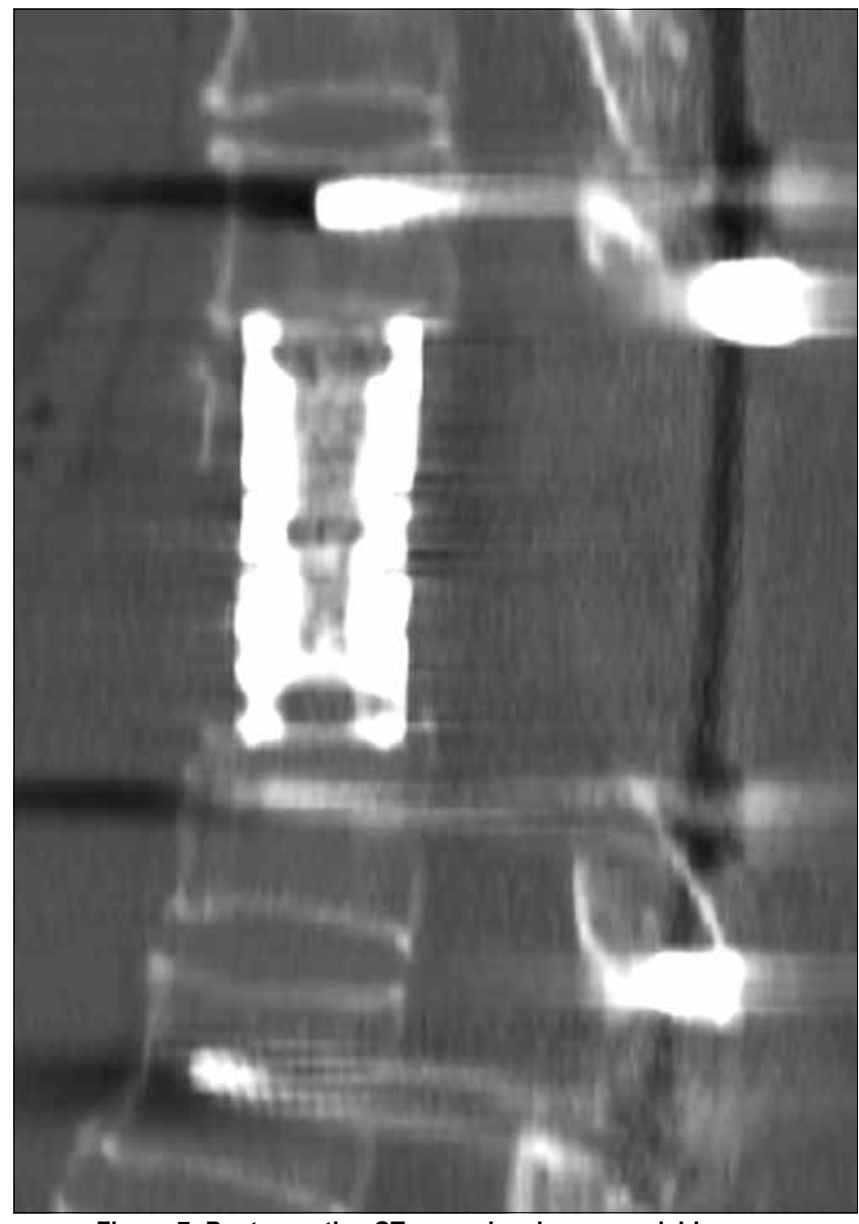

Figure 7: Postoperative CT scan showing expandable cage reconstruction and endplate 
In the case of costotransversectomy, this rate is relatively low, and new technique of endoscopic assistance may reduce this complication rate further. ${ }^{[1,11]}$ However, in the case of the lateral extracavitary approach, the pulmonary complication rate is as high as $55 \% .{ }^{[12]}$ In contrast, we used a rib disarticulation technique without pleural dissection to separate the rib from the vertebral body. This allows for mobilization of the chest wall to place an expandable cage from a posterior approach. Because the rib is disarticulated and not resected, there is less risk of injury to the segmental vessel as it courses lateral to the vertebral body and the pleura. Although ligation of the nerve roots will still place the spinal cord at susceptibility for ischemic injury, the preservation of the rib head provides a barrier between the operative field and the segmental vessel. Moreover, this technique allows for multiple levels of corpectomies to be performed from a posterior approach because it affords large, expandable cage reconstruction for solid anterior column support.

Much of the early experience of anterior column restoration used either structural allograft or methylmethacrylate (MMA) (with or without stabilizing Steinman pins) for reconstruction. . $^{[2,4,5,13-15]}$ MMA was especially useful because it could be injected to the anterior column through the limited space from the posterior spine. While MMA and structural allograft offer adequate anterior column support, they do not offer height restoration and kyphosis correction by themselves. Moreover, for secure wedging of nonexpandable grafts, compression must be applied posteriorly after anterior interbody reconstruction.

Only a few authors have used titanium cages for reconstruction of the ventral spine when a posterior approach is used. Most of the early experience with titanium cages was with the nonexpandable cages, which tend to be smaller than the expandable cages. Wiggins et al. described the largest series with 29 patients having tumors of the thoracic spine. ${ }^{[1]}$

The authors used costotransversectomies and either allograft bone or a MMA-filled titanium mesh cages. ${ }^{[1]}$ Dvorak et al. reported a multilevel reconstruction of the ventral spine with a nonexpandable cage via a posterior approach. ${ }^{[16]}$

The recent development of expandable cages has been proven to be especially useful for height restoration and kyposis correction. ${ }^{[17]}$ However, because of the size of many titianum expandable cages, its placement through a posterior approach has been limited, and only a few authors have written about using expandable cages through a posterior approach.

Snell et al. reported 15 patients who underwent a single-stage posterior approach for vertebrectomy with anterior cage placement. ${ }^{[18]}$ However, expandable cages were successfully used in only two patients, and there were no multilevel reconstructions in this series. ${ }^{[18]}$ Hunt et al. describe a technique of placing an expandable cage into the lumbar spine but this is at the level of the lumbar spine and the rib heads are not an issue. ${ }^{[19]}$

\section{Conclusion}

Multilevel thoracic corpectomies can be performed via a posterior transpedicular approach without costotransversectomy or lateral extracavitary approaches. Rib head disarticulation can facilitate placement of larger expandable cages while decreasing the risk of pleural injury.

\section{References}

1. Wiggins GC, Mirza S, Bellabarba C, West GA, Chapman JR, Shaffrey CI. Perioperative complications with costotransversectomy and anterior approaches to thoracic and thoracolumbar tumors. Neurosurg Focus 2001;11:e4.

2. Akeyson EW, McCutcheon IE. Single-stage posterior vertebrectomy and replacement combined with posterior instrumentation for spinal metastasis. J Neurosurg 1996;85:211-20.

3. Bassewitz HL. Principles and Practice of Spine Surgery. In: Vaccaro AR, Betz RR, Zeidman SM, editors. Philadelphia PA: Mosby Publishing Company; 2003.

4. Bilsky MH, Boland P, Lis E, Raizer JJ, Healey JH. Single-stage posterolateral transpedicle approach for spondylectomy, epidural decompression, and circumferential fusion of spinal metastases. Spine 2000;25:2240-9, discussion 250.

5. Cahill DW, Kumar R. Palliative subtotal vertebrectomy with anterior and posterior reconstruction via a single posterior approach. J Neurosurg 1999;90:42-7.

6. Cybulski GR, Stone JL, Opesanmi O. Spinal cord decompression via a modified costotransversectomy approach combined with posterior instrumentation for management of metastatic neoplasms of the thoracic spine. Surg Neurol 1991;35:280-5.

7. Das K, Rawanduzy A, Couldwell WT. Posterolateral approach for decompression with anterior and posterior fusion: A less invasive surgical technique for stabilization of the thoracic spine. Case report. Neurosurg Focus 1998;4:e3.

8. Smith JT, Gollogly S, Dunn HK. Simultaneous anterior-posterior approach through a costotransversectomy for the treatment of congenital kyphosis and acquired kyphoscoliotic deformities. J Bone Joint Surg Am 2005;87:2281-9.

9. Steinmetz MP, Mekhail A, Benzel EC. Management of metastatic tumors of the spine: Strategies and operative indications. Neurosurg Focus 2001;11:e2.

10. Shaw B, Mansfield FL, Borges L. One-stage posterolateral decompression and stabilization for primary and metastatic vertebral tumors in the thoracic and lumbar spine. J Neurosurg 1989;70:405-10.

11. McLain RF. Spinal cord decompression: An endoscopically assisted approach for metastatic tumors. Spinal Cord 2001;39:482-7.

12. Resnick DK, Benzel EC. Lateral extracavitary approach for thoracic and thoracolumbar spine trauma: Operative complications. Neurosurgery 1998;43:796-802; discussion 802-3.

13. El-Meshtawy M. Posterolateral Corpectomy of Thoracolumbar Fractures: Results of 100 Cases. (Abstract) Presented at $7^{\text {th }}$ International Congress of Spinal Surgery. Eur Spine J 2005;14:298-326.

14. Rath SA, Neff U, Schneider O, Richter HP. Neurosurgical management of thoracic and lumbar vertebral osteomyelitis and discitis in adults: A review of 43 consecutive surgically treated patients. Neurosurgery 1996;38:926-33. 
15. Wang JC, Boland P, Mitra N, Yamada Y, Lis E, Stubblefield M, et al. Single-stage posterolateral transpedicular approach for resection of epidural metastatic spine tumors involving the vertebral body with circumferential reconstruction: Results in 140 patients. Invited submission from the Joint Section Meeting on Disorders of the Spine and Peripheral Nerves, March 2004. J Neurosurg Spine 2004;1:287-98.

16. Dvorak MF, Kwon BK, Fisher CG, Eiserloh HL $3^{\text {rd }}$, Boyd M, Wing PC. et al. Effectiveness of titanium mesh cylindrical cages in anterior column reconstruction after thoracic and lumbar vertebral body resection. Spine 2003;28:902-8

17. Thongtrangan I, Balabhadra RS, Le H, Park J, Kim DH. Vertebral body replacement with an expandable cage for reconstruction after spinal tumor resection. Neurosurg Focus 2003;15:E8.

18. Snell BE, Nasr FF, Wolfla CE. Single-stage thoracolumbar Vertebrectomy with circumferential reconstruction and arthrodesis: Surgical technique and results in 15 patients. Neurosurgery 2006;58:ONS-263-8; discussion ONS-269.

19. Hunt T, Shen FH, Arlet V. Expandable cage placement via a posterolateral approach in lumbar spine reconstructions. Technical note. J Neurosurg Spine 2006;5:271-4.

Accepted on 06-03-2009

Source of Support: Nil, Conflict of Interest: None declared.

\section{Forthcoming Events}

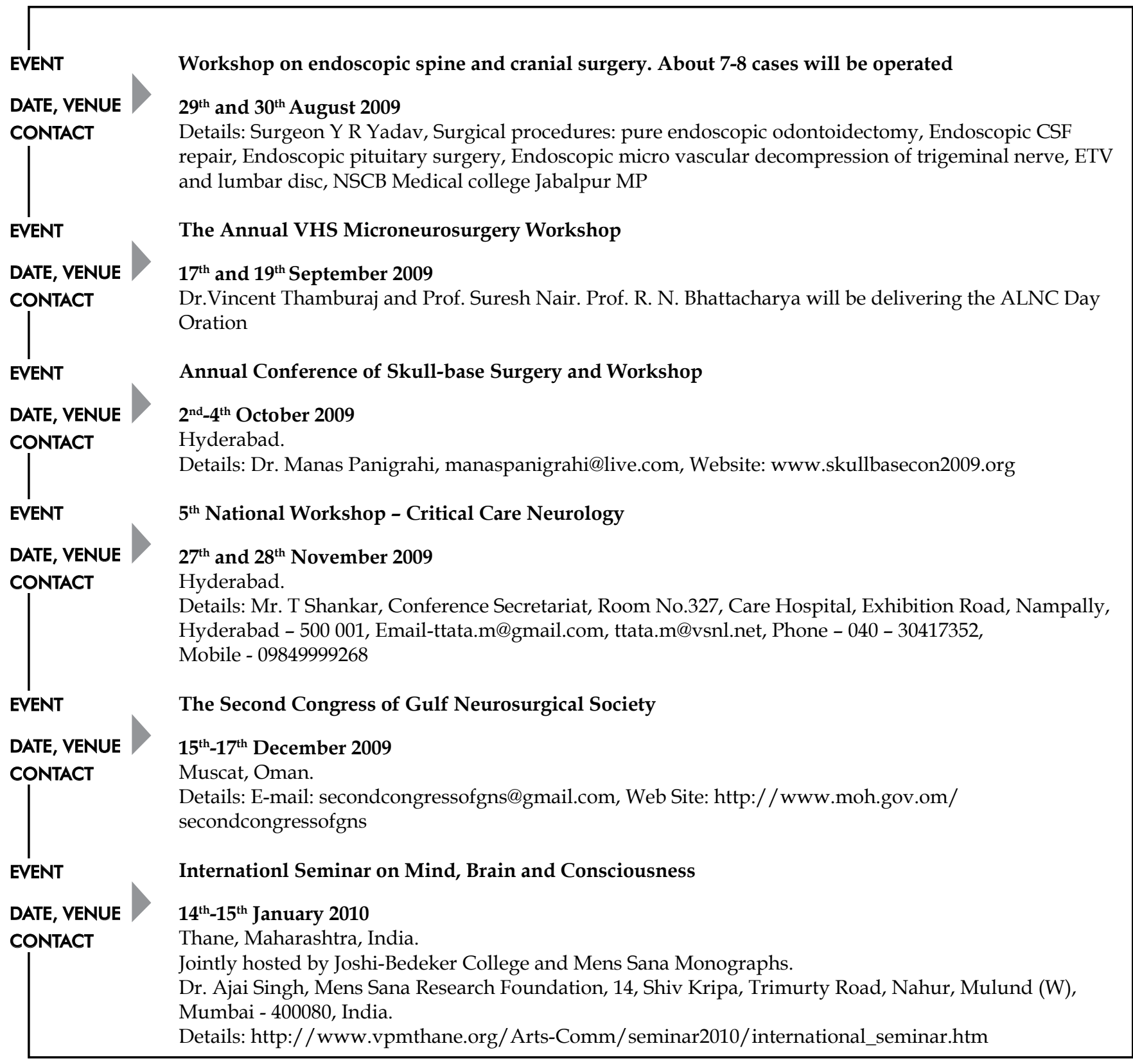

